\title{
Multirate Digital Signal Processing for Image Reconstruction using Error Optimization Techniques: The Combined Adaptive Weiner Filter and the MDBUTMF
}

\author{
Winston Tumps Ireeta \\ Department of Physics \\ Makerere University
}

\author{
Achire Tito \\ Department of Physics \\ Makerere University
}

\author{
Yusto Kaahwa \\ Department of Physics \\ Makerere University
}

\begin{abstract}
In this study, reconstruction of clearer images using error optimization techniques was done. Minimization of noise from the image and the study of the performance of a combination of the Weiner Filter (WF) via the Least Mean Square (LMS) Algorithm and the Modified Decision Based Unsymmetrical Trim Median Filter (MDBUTMF). MATLAB 7.11.0 (R2010b) as a digital signal processing tool was used. The findings reveal that the WF-LMS algorithm fails to remove salt noise (zero valued pixels). These findings turn out to be a potential contribution of this study to the field of DSP in computational physics and optics. Several studies in multirate digital signal processing have not pointed this anomaly of WF-LMS algorithm in their work nor has it been published by any publishing institutions to the best of our knowledge. MDBUTMF performance was found to be better than WF-LMS at high noise variances. However, observations show that the resolution of the image with MDBUTMF decreases with increasing noise variances (NV). The results of the proposed method (a combination of WF-LMS and MDBUTMF) were found to be generally effective and superior compared to WF-LMS and MDBUTMF when used separately. The method therefore makes a potential contribution in improving restoration and visibility of images. For qualitative evaluation and measurement, the Mean Average Error (MAE), Mean Square Error (MSE), Peak Signal to Noise Ratio (PSNR) and Signal to Noise Ratio (SNR) were used and the effectiveness of the filters increases in the order of WF-LMS, MDBUTMF and the proposed method. It is concluded that the generality that WF-LMS denoises salt and pepper noise no longer applies. It is also concluded that the proposed method is superior compared to the WF-LMS and the MDBUTMF when used separately. It is then recommended that WF-LMS needs modification to denoise the salt noise and thus the proposed method of combination of WF-LMS and the MDBUTMF.
\end{abstract}

\section{General Terms}

Mean Square Error, Modified Decision Based Unsymmetrical Trim Median Filter (MDBUTMF), Weiner Filter via the Least Mean Square and Peak Signal to Noise Ratio.

\section{Keywords}

Image Reconstruction; Weiner Filter; Signal Processing; Least Mean Square; Signal to Noise Ratio.

\section{INTRODUCTION}

The process of removing noise from the original image is still a demanding problem for most researchers [1] and [2]. Visual information transmission is very valuable today. People prefer still images to a thousand of words [1]. However, noises do accompany signals during their creation, transformation and transmission and this affects image visibility variably and imposes hardship to its scientific interpretation and analysis [2].

Thus far, when an image is acquired by any imaging systems, often the vision system for which it is intended fails to use it directly. It may be corrupted by random variations in intensity, illumination, poor contrast and impulse noise introduced in the system. Such problems call for digital image enhancement techniques [1] and [3].

This paper presents some tools for digital image signal processing and reconstruction. Basically, in this paper, error minimization techniques were employed where competing and unwanted signals such as impulse noises and in particular salt and pepper noises are removed from the desired signal. Many researches and industries have attached value to high image resolution and visibility; Remote Sensing, Medical Imaging, Non-destructive Evaluation, Forensic Studies, Military, Film Industry, Document processing and Printing Industry require improvement in the area of digital image processing [4].

Digital Signal Processing and image reconstruction in the above areas have been made simpler by the development of multirate system theory [5]. Multirate signal processing theory deals with analysis of a system comprised of multiple signals at different sampling rates. The theory has traditionally been applied to the contexts of filter banks [6]. This plays a very important role in signal decomposition, analysis and reconstruction [7].

In reconstructing images, several approaches were developed [8]. The most widely used is the filtering approach. This paper employed optimal observation model using WF-LMS together with the MDBUTMF. The model uses Least Mean Square Error (LMSE) method to optimize errors in the signals and multirate system can be applied to it. This method is not extensively used in digital image processing because of the mathematical complexity involved in calculating the filter coefficients desired for image reconstruction [4]. WF-LMS is used for de-noising a set of input signals that are degraded by salt and pepper noise. It is a class of optimum linear filters [9], [10] and [11]. The design of the filter is distinct. It is based on a statistical approach and is optimal in the sense of Minimum Mean Square Error (MMSE) [12].

However, WF-LMS is ineffective at high noise variances [8]. The MDBUTMF was proposed to de-noise salt and pepper noise introduced in an image [13]. In the MDBUTMF algorithm, the noisy image is de-noised by a $3 \mathrm{X} 3$ window elements and then pixels are arranged in increasing or 
decreasing order. Then the pixel values ' 0 's and '255's in the image (i.e., the pixel values responsible for the salt and pepper noise) are removed and the median value of the remaining pixels is used to replace the noisy pixel. This algorithm was found to be ineffective in de-noising salt $\&$ pepper noise at high noise variances ranging from $70 \%$ to $95 \%$ [14].

It is noted that the Adaptive WF-LMS does not give a better result even at low noise variances and the MDBUTMF does not give a better result at salt and pepper noise variances ranging from $70 \%$ to $95 \%$. Therefore, a combination of the Adaptive WF-LMS and the MDBUTMF (WFLMSMDBUTMF) algorithm is proposed in this study for improved image restoration and visibility. The principle behind the selection of these filters is not to use the already best performing filters but to improve image visibility using an appropriate combined method.

\section{SALT AND PEPPER NOISE ON IMAGE, VIDEO SIGNAL AND AUDIO}

The salt and pepper noise cover the surface of image with black and white spots. Conventionally, 0 's are responsible for the black while 255 's values are responsible for the white spots on the images [15]. In video signals, it causes ripples on the television screen [15]. This makes images in television screen distorted and undesirable to human visual system. In audio channel, they cause odd sound that distorts the original messages being conveyed to the listeners. This study is concerned with salt and pepper noise removal from images.

Various kinds of noises exist in an image and a variety of denoising techniques have been proposed by different researchers [16]. Gaussian, speckle, salt \& pepper, shot noises are types of noises that are present in an image. The principle approach of image de-noising is filtering. The filtering techniques used are median, Gaussian, average and Weiner Filtering. The resulting image from these approaches is either blurred or over smoothed due to losses. In this paper a new approach of combining WF-LMS and MDBUTMF is proposed.

\section{DIGITAL IMAGE \\ REPRESENTATION, COORDINATE CONVENTION AND MATRIX REPRESENTATION}

Equation 1 is the conventional representation of the images to be considered. The image information was then represented in matrix form as shown in equation 2 .

The image information generated above was stored in gray scale coding scheme for computational efficiency. It represents the brightness of the image at that point [17].

$f(x, y)=\left[\begin{array}{lllc}f(0,0) & f(0,1) & \ldots & f(0, N-1) \\ f(1,0) & f(1,1) & \ldots & f(1, N-1) \\ \vdots & \vdots & \ldots & \vdots \\ f(M-1,0) & f(M-1,1) & \ldots & f(M-1, N-1)\end{array}\right]$

$$
f(x, y)=\left[\begin{array}{lccc}
f(1,1) & f(1,2) & \ldots & f(1, N) \\
f(2,1) & f(2,2) & \ldots & f(2, N) \\
\vdots & \vdots & \ldots & \vdots \\
f(M, 1) & f(M, 2) & \ldots & f(M, N)
\end{array}\right]
$$

A linear adaptive WF-LMS with the noisy data as input was designed and the requirement of minimizing the effect of the noise at the filter output was considered as shown in Figure 1 [18]. The filter coefficients are modified by a feedback process designed to make the filters' output $y[n]$ close to the desired signal $d[n]$ by minimizing the error $e[n]$. The filter output, the error and filter coefficients were calculated adaptively using the LMS algorithm in equations;

$$
\begin{aligned}
& y(i, n)=x(n) * h(i, n) \\
& e[i, n]=d[n]-y[n] \\
& h_{n}[i, n+1]=h_{n}[i, n]+\Delta e[i, n] * x[n] \\
& \text { where; } \\
& x[n] \text { is the noisy signal } \\
& e[n] \text { is the error } \\
& d[n] \text { is the original signal and } \Delta \text { is the convergent factor. }
\end{aligned}
$$

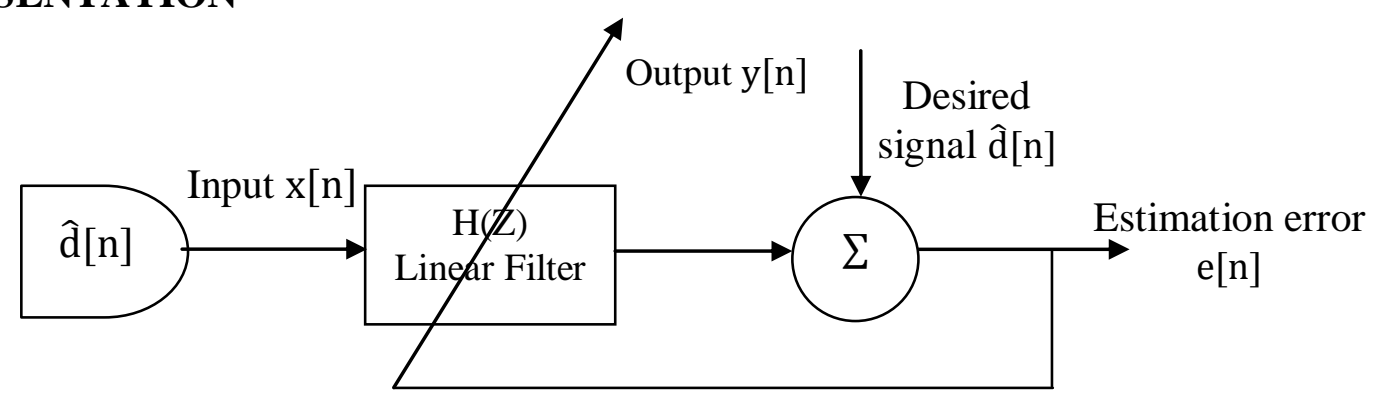

Figure 1: Adaptive Filter [18]

The filtering process described above starting from image acquisition, data class and intensity coding, signal decomposition and decimation, translation and computation of filter coefficients are summarized in Figure 2. 
(1) Image

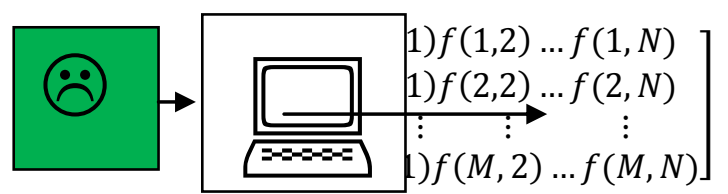

(3) Image transformed to gray

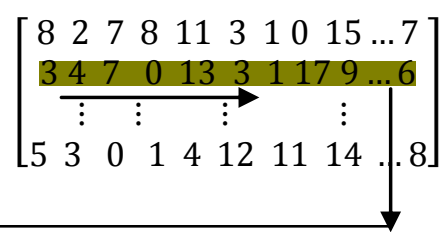

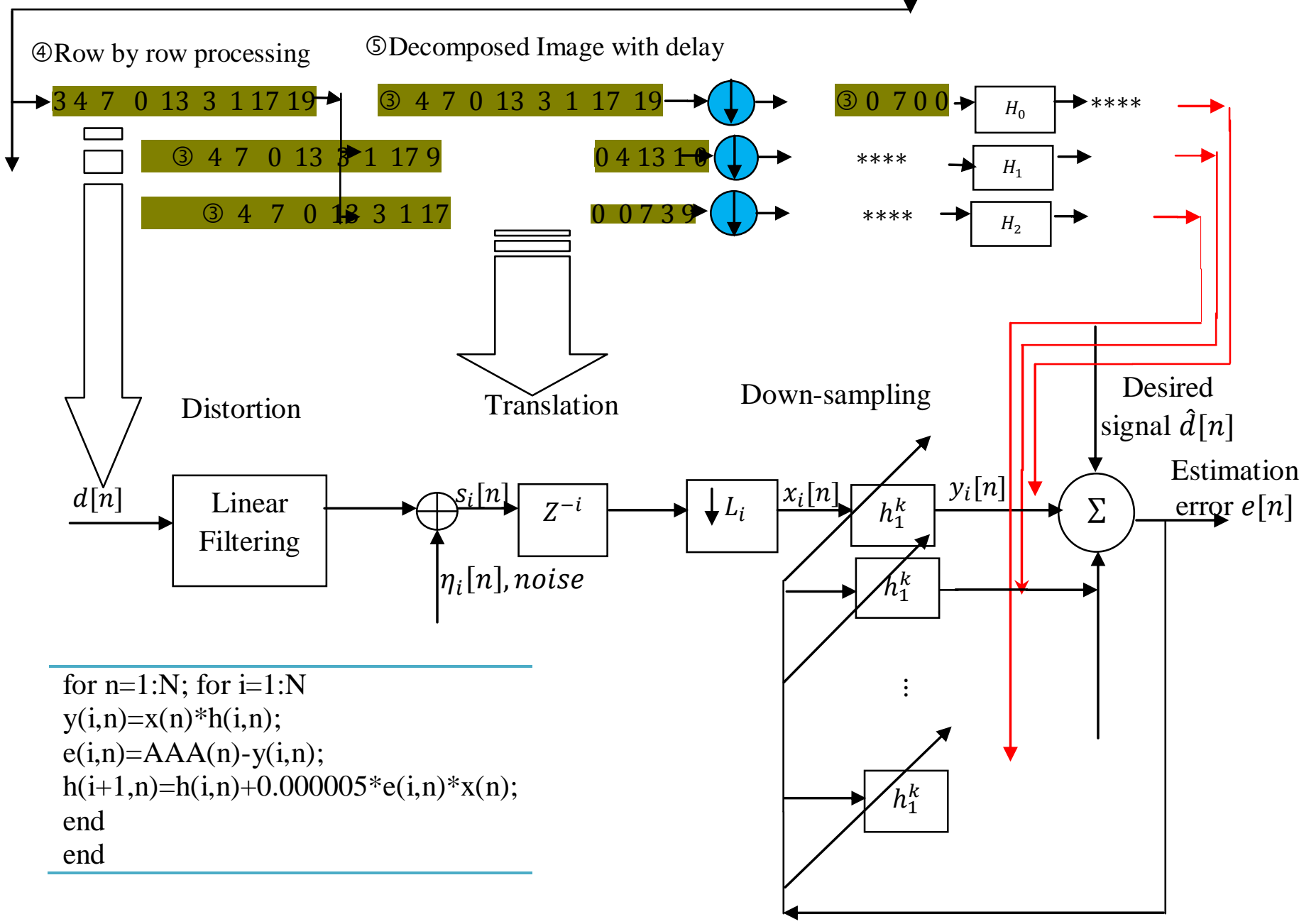

Figure 2: Adaptive Wiener Filter illustration design for image de-noising

The LMS algorithm suggests that for iteration number, N; $\mathrm{y}(\mathrm{i}, \mathrm{n})$ is the desired signal output, $\mathrm{AAA}(\mathrm{n})$ is the undistorted signal input, $e(i, n)$ is the signal error to be optimized when the filter updates itself to a desired value and $h(i, n)$ is the filter coefficient that keeps updating by a feedback process until the error is minimized. In a multichannel representation and for periodically time varying signals, the estimate can be written as presented by Semmlow et al. [19].

\section{MODIFIED DECISION BASED UNSYMMETRICAL TRIM MEDIAN FILTER (MDBUTMF)}

This algorithm was proposed by Chaitanya [16] and is proposed in this paper to be used in combination with the Adaptive WF-LMS. Its operation is described below.

1. The pixels inside the window are sorted out in ascending order.

2. Min, max and median of the pixel values in the processing window are determined.

3. The algorithm reads the noisy image, $p(i, j)$ which is taken as a processing pixel.

4. If the central pixel lies between minimum and maximum values, then it is detected as uncorrupted pixel and is left undisturbed. Otherwise, it is considered as a corrupted pixel.

5. If the processing pixel is detected as corrupted, a $3 \times 3$ window is taken around the pixel.

6. If the processing window contains all minimum or maximum pixel elements only, then the processing pixel was replaced by the second element above or below it.

7. If the processing window contains both minimum and maximum pixel values, then the processing pixel is replaced by the mean of the elements in the window

8. If the processing window contains not only the min or max value, the minimum and the maximum are trimmed 
out and the processing pixel was replaced by the mean of the remaining elements in the window. Then the window is moved to a new set of values, with the next pixel to be processed at the center and steps 1-8 is repeated as shown in Figure 3.

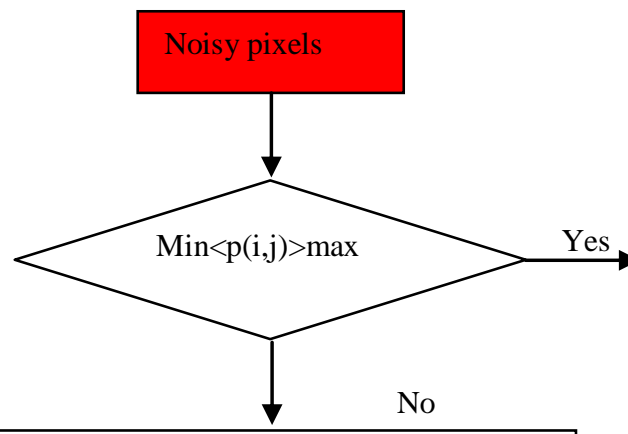

Select a 2D $3 X 3$ window (w) with centre $\mathrm{p}(\mathrm{i}, \mathrm{j})$ as a processing pixel

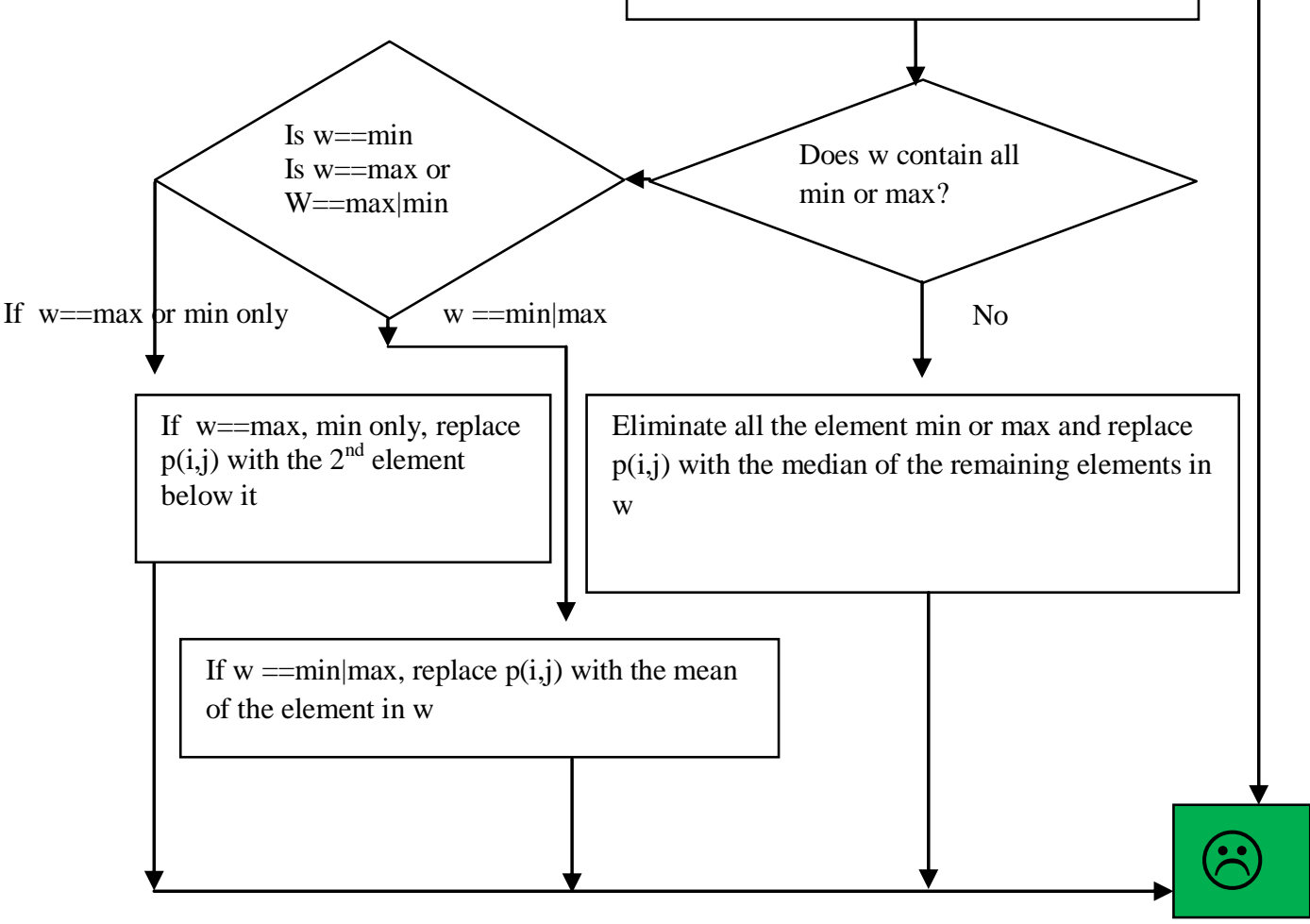

Figure 3: Modified Decision Based Unsymmetrical Trim Median Filter (MDBUTMG) [13]

The algorithm in figure 3 was translated into an executable MATLAB program and its functionality was tested first on a data matrix and an image sample. Later the proposed method, that is, a combination of WF-LMS in Figure 2 and the MDBUTMF in Figure 3 was considered. 


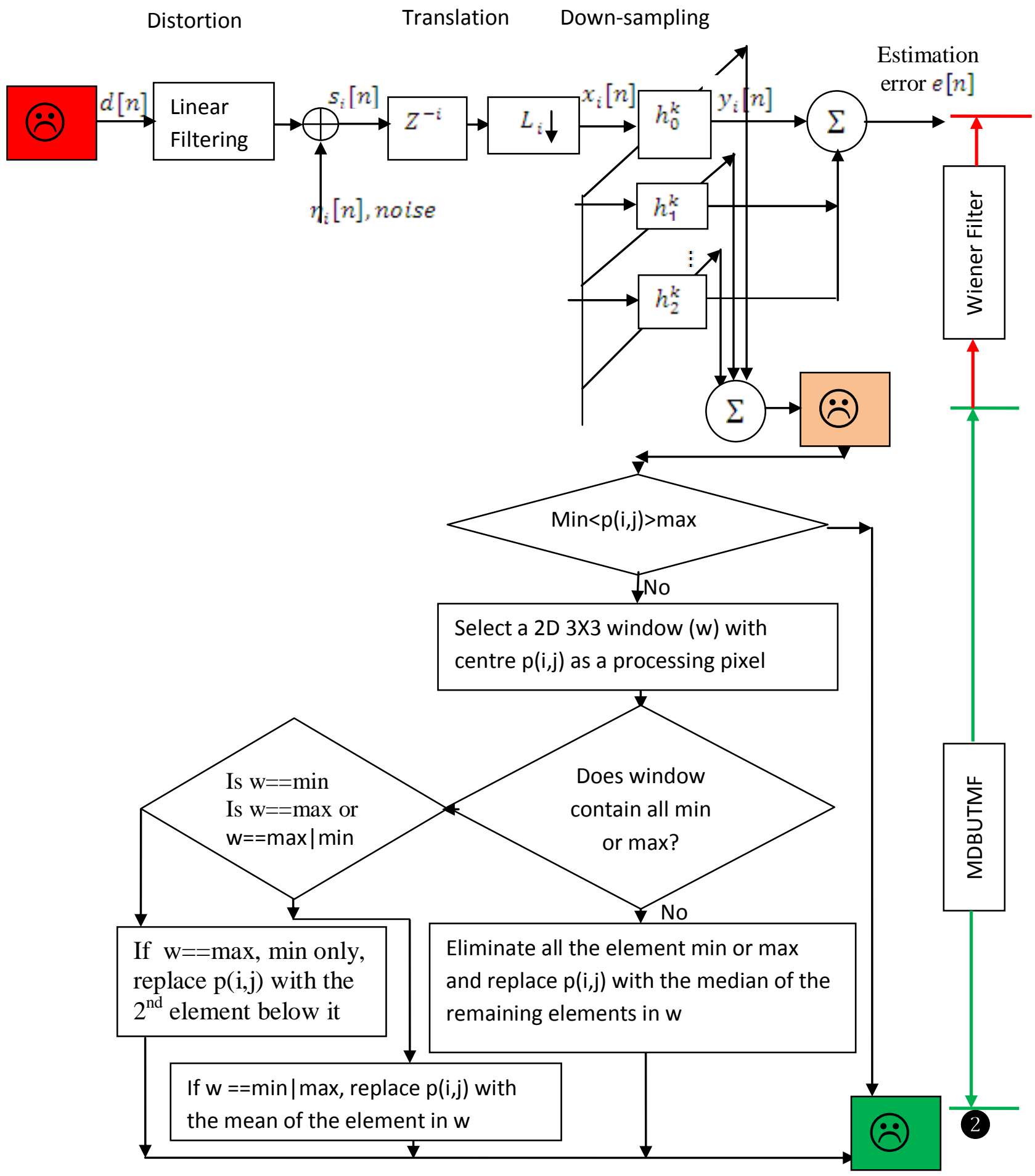

Figure 4: Proposed Weiner Filter-Modified Decision based Unsymmetrical Median Filter

As can be seen, the proposed method has two stages. In the first stage, the filtering of the noisy image is done by WFLMS. This results into image which is still noisy because of the Wiener Filter's failure to de-noise salt and pepper noise at high noise variances. In the second step, the MDBUTMF picks up the pixels generated by the WF-LMS and then output image, which is clearer, is obtained.

\section{QUANTITATIVE AND QUALITATIVE EVALUATION}

For Image Quality Measurement, both the subjective and the objective measurement approaches were used [20] The subjective quality measurements use human experts' opinion and image quality while the objective measurements are performed using mathematical algorithms. The four main objective image quality measures used in this paper were the Mean Average Error (MAE), the Mean Square Error (MSE), the Peak Signal to Noise Ratio (PSNR) and the Signal to 
Noise Ratio (SNR).

$$
\mathrm{MAE}=\frac{1}{M N} \sum_{i=1}^{M} \sum_{j=1}^{N}|x(i, j)-\hat{x}(i, j)|
$$

where;

$$
\begin{aligned}
& x(i, j) \text { is original pixel value } \\
& \hat{x}(i, j) \text { is the corrupted signal } \\
& \mathrm{M} \text { and } \mathrm{N} \text { are the image signal dimensions }
\end{aligned}
$$

$$
\mathrm{MSE}=\frac{1}{M N} \sum_{i=1}^{M} \sum_{j=1}^{N}[x(i, j)-\hat{x}(i, j)]^{2}
$$

$$
\operatorname{PSNR}=10 \log _{10}\left(\frac{L^{2}}{\mathrm{MSE}}\right)
$$

$$
\mathrm{SNR}=10 \log _{10} \frac{\sum\left(x^{2}\right)}{\sum\left(e^{2}\right)}
$$

\section{RESULTS}

The response of the WF-LMS to different salt and pepper noise variances was observed. A few rows of the image pixel were extracted, $40 \%$ of salt and pepper noise was added to it. The results show that the WF-LMS is very effective to reconstruct the pixel values replaced by pepper noise, altered due to decimation and translation and those that remained unaffected (table 1). A significant finding shows that WFLMS does not de-noise black coloured noise (zero valued) as indicated in table 1 . The evaluation results show that if the selected corrupted signal, $x[n]$, is zero valued, the estimated signal outputs are all zeros and the signal error $\mathrm{e}[\mathrm{i}, \mathrm{n}]$ equals the original signal value, AAA[n]. Since $x[n]$ and the first filter coefficient are zeros, all the updated filter coefficients are also zeros. For example, the noisy signal $x[1]$, that is, pixel replaced with salt noise (zero valued signal) as in table 1 was selected and the output $y[\mathrm{i}]$ and $\mathrm{h}[\mathrm{i}]$ are zeros respectively for all the iterations. This algorithm supports the finding that WF via LMS does not effectively remove salt and pepper noise at high noise variances.

The optimal model in figure 3 was applied to minimize the error. Table 1 shows that WF-LMS removes white colored noise (255) and minimizes error due to translation and decimation. For example, for $\mathrm{x}[6]$ the real pixel value is 146 but the corrupted signal has 138 as its pixel value. This is not the effect of the salt and pepper noise but rather the effect of decimation and translation on the signal. A perfect reconstruction of 145.5837 closure to 146 (the original pixel value) was achieved. To achieve this nearly perfect value, the filter adjusts its coefficient $\mathrm{N}+1$ times as shown in table 1 until it reaches 1.0474. From table 1, it can be seen that the error is zero. This result matches the requirement of optimal filtering only for the pixel values replaced by pepper noise, altered due to decimation and translation and those remain unaffected.

Table 1: Operation of Adaptive Weiner Filter in optimal process

\begin{tabular}{|l|r|r|r|r|r|r|r|}
\hline & \multicolumn{1}{|l|}{$\begin{array}{l}\text { Pixel } \\
\text { replaced by } \\
\text { salt noise }\end{array}$} & \multicolumn{2}{|l|}{$\begin{array}{l}\text { Pixel } \\
\text { Unaffected }\end{array}$} & \multicolumn{2}{l|}{$\begin{array}{l}\text { Pixels Replaced by Pepper } \\
\text { Noise }\end{array}$} & \multicolumn{2}{l|}{$\begin{array}{l}\text { Pixel Changed due } \\
\text { to Decimation and } \\
\text { translation }\end{array}$} \\
\hline $\begin{array}{l}\text { Original Pixel } \\
\text { values }\end{array}$ & 129.0000 & 111.0000 & 120.0000 & 128.0000 & 150.0000 & 146.0000 & 129.0000 \\
\hline Noisy Pixels & 0.0000 & 111.0000 & 255.0000 & 255.0000 & 255.0000 & 139.0000 & 138.0000 \\
\hline $\begin{array}{l}\text { Filter } \\
\text { coefficients }\end{array}$ & 0.0000 & 0.9957 & 0.4692 & 0.5011 & 0.5865 & 1.0474 & 0.9315 \\
\hline Denoised pixels & 0.0000 & 110.5194 & 119.646 & 127.7741 & 149.5575 & 145.5837 & 128.5470 \\
\hline Error & 129.0000 & 0.0000 & 0.0000 & 0.0000 & 0.0000 & 0.0000 & 0.0000 \\
\hline
\end{tabular}

It can be concluded from the table that;

I. The algorithm does not meet the optical requirement for perfect image reconstruction. The optimal filtering condition is only true for the pixel values replaced by pepper noise, altered due to decimation and translation and those remain unaffected. For pixel value replaced by salt noise, the optical requirement does not apply.

II. The errors for the pixel values replaced by pepper noise, altered due to decimation and translation and those remain unaffected are minimized by WF-LMS algorithm while the error for the pixel values affected by salt noise are unnecessorily big.

III. Filter coeeficients for the pixel values replaced by pepper noise, altered due to decimation and translation and those that remain unaffected update themselves until they reach the desired values and the errors are minimized to zeros. The filter coefficients for the pixel values affected by salt noise do not update themselves. They remain as the initial filter values (zero's).

To observe this anomaly in the WF-LMS, the filters coefficients and error terms in table 1 were plotted against iteration number N. From figure 4 , it can be seen that as the filter updates its coefficients, the errors keep reducing until they converge to zero. However, for the pixels values replaced with salt noises, the filter does not update its coefficients as shown. The explanation to this problem is mathematically expressed in table 1 . The error terms for the pixel replaced by salt noise also remain constant at original pixel values as shown in table 1 and in figure 4. 


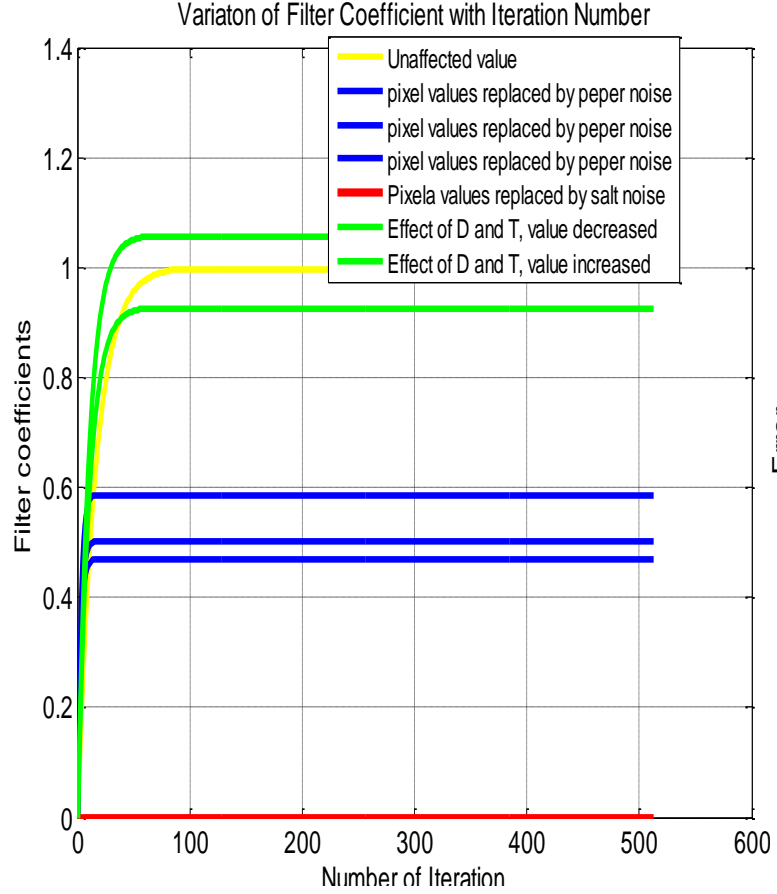

(a)

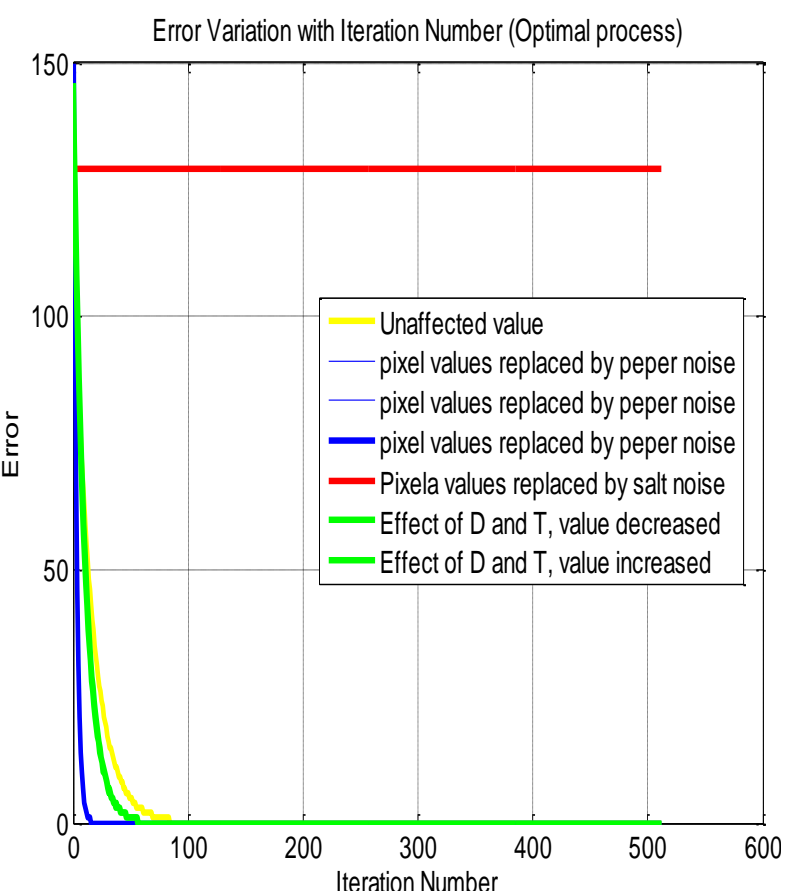

(b)

Figure 4: Convergence of square error in optimal process: (a) variation of updated filter coefficient with iteration number and (b) variation of error with iteration number in optimal process

Where the value decreased, it increased the mean, the decimation (D) and translation (T) resulting into the pixel value getting lower or higher than the original pixel value (See table 1).

From figure 4, the study established that the WF-LMS algorithm cannot achieve perfect image reconstruction. The algorithm satisfies the optimal condition selectively. It is this selective response of WF-LMS algorithm to salt and pepper noise values that makes the filter vulnerable to de-noise salt and pepper noise even at low noise variances as shown in figure 4(b).

\subsection{MDBUTMF Algorithm and its Response to Salt and Pepper Noise}

$\begin{array}{lllclcclcll}0 & 0 & 0 & 0 & 0 & 0 & 0 & 0 & 0 & 0 & 0 \\ 0 & 0 & 0 & 0 & 0 & 0 & 0 & 0 & 0 & 0 & 0 \\ 0 & 0 & 63 & 55 & 85 & 0 & 0 & 0 & 255 & 0 & 0 \\ 0 & 0 & 255 & 65 & 85 & 0 & 0 & 0 & 255 & 0 & 0 \\ 0 & 0 & 55 & 255 & 93 & 71 & 66 & 98 & 54 & 0 & 0 \\ 0 & 0 & 21 & 65 & 0 & 33 & 21 & 241 & 98 & 0 & 0 \\ 0 & 0 & 61 & 90 & 80 & 48 & 50 & 69 & 220 & 0 & 0 \\ 0 & 0 & 211 & 97 & 112 & 91 & 73 & 0 & 87 & 0 & 0 \\ 0 & 0 & 56 & 0 & 78 & 211 & 195 & 87 & 0 & 0 & 0 \\ 0 & 0 & 0 & 0 & 0 & 0 & 0 & 0 & 0 & 0 & 0 \\ 0 & 0 & 0 & 0 & 0 & 0 & 0 & 0 & 0 & 0 & 0\end{array}$

(a)

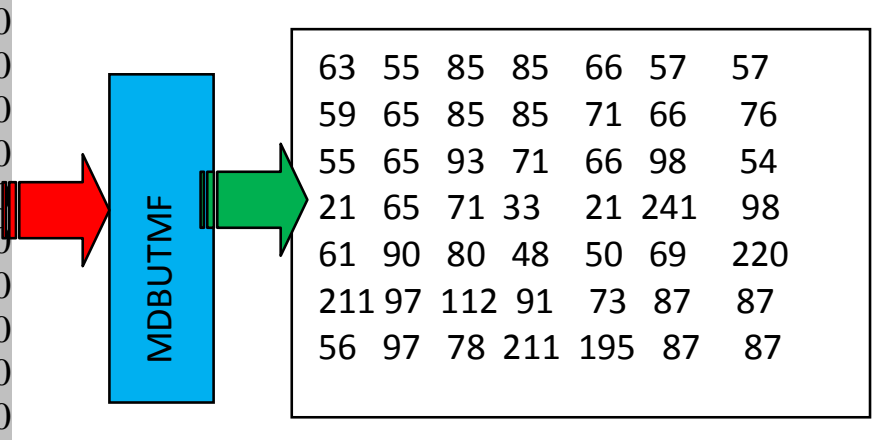

(b)

Figure 5: Output of MDBUTMF: (a) Noisy date matrix (b) De-noised data matrix

The significant application of MDBUTMF to the proposed algorithm is that it eliminates even the salt noises which are not possible to be removed by the WE-LMS as was shown in table 1. Its major diadvantage however is that it cannnot restore the original pixel values that have changed value due to decimation and translation. The argument being that the pixels that have changed their values due to decimation and translation are not always salt (zero valued) or pepper noise (255 valued) as shown in figure 5 .

Since MDBUTMF is designed to detect and de-noise salt and pepper noise and leave the other pixel values unaffected, it would take the pixels altered due to decimation and translation as the true pixel values which is not. For this reason, it is not a good practice to impelment MDBUTMF algorithm on decimated and translated signals. However, this problem is sorted out by implementing WF-LMS algorithm first. Therefore a combination of WF-LMS and the MDBUTMF is expected to give better results.

After testing the algorithms on the test image and the data matrix, full image reconstruction was considered. 


\subsection{Application to Makerere University Main Building}

To observe the effect of Wiener Filter, the MDBUTMF and the proposed method on a real image, a portion of an image (extracted from Makerere University main building) shown in figure 6 was used. Figure 6(a) shows the original image to which salt and pepper noise of different noise variances was added (figures 6(b)-6(d)). The resultant signals were decimated with a decimation factor, $\mathrm{L}=3$.

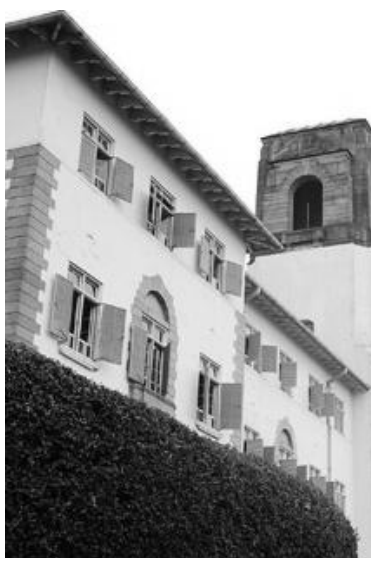

(a)

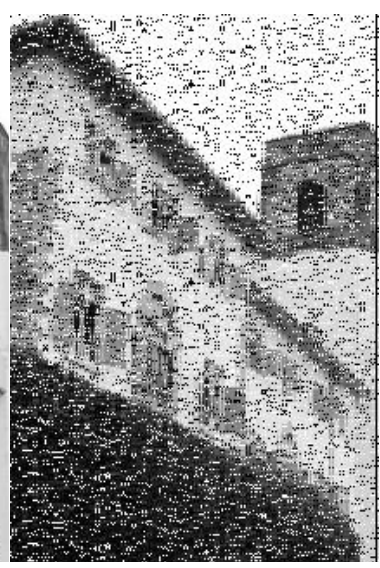

(b)

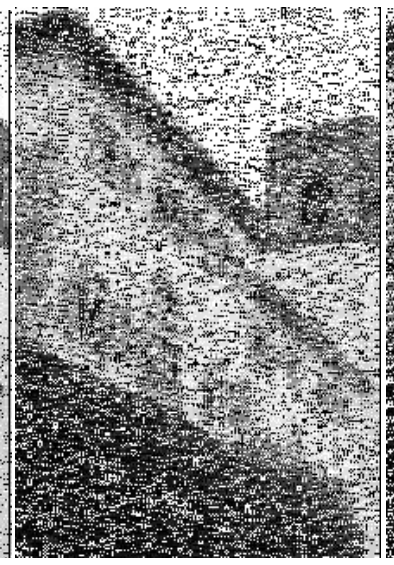

(c)

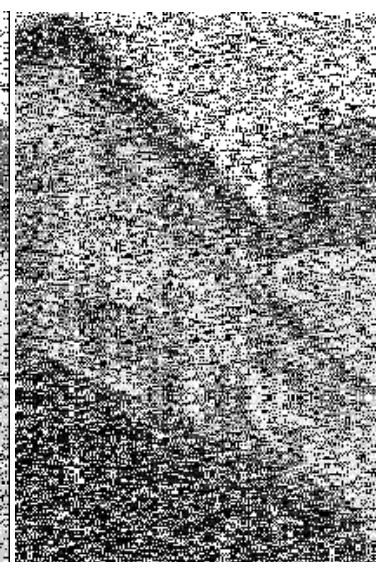

(d)

Figure 6: Effect of Salt and Pepper Noise on Makerere University Image (a) the Original image, (b) the image blurred with $20 \%$ salt and pepper noise, (c) the image blurred with $40 \%$ salt and pepper noise, (c) the image blurred with $60 \%$ salt and pepper noise

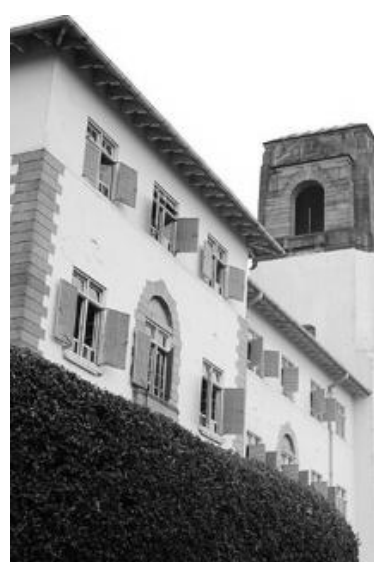

(a)

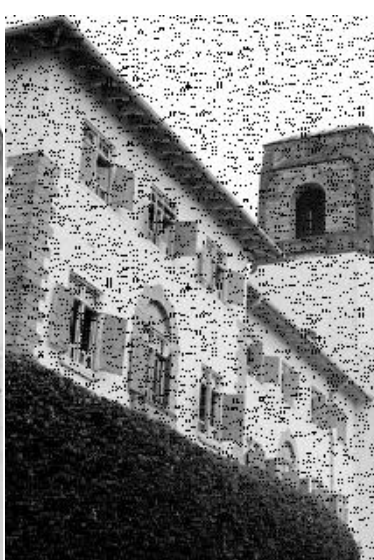

(b)

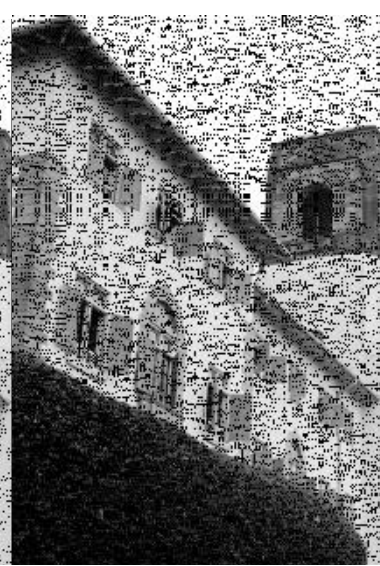

(c)

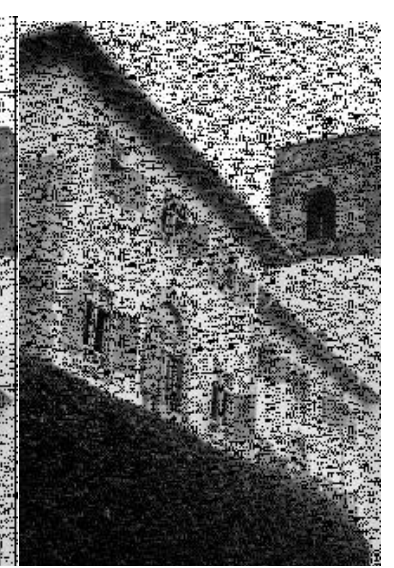

(d)

Figure 7: Outcome of WF-LMS algorithm in de-noising salt and pepper noise from Makerere University (a) Original image, (b) outcome of WF for $20 \%$ salt and pepper noise, (c) outcome of WF for $40 \%$ salt and pepper noise, (d) outcome of WF for $60 \%$ salt and pepper noise

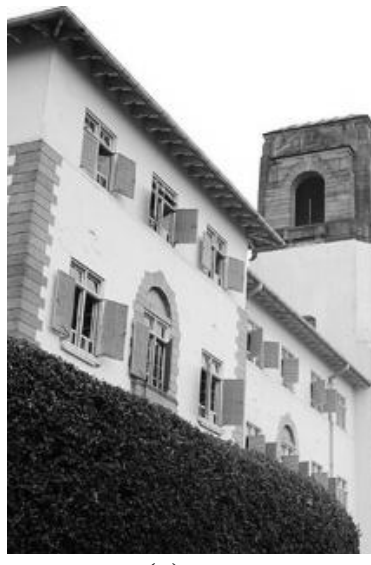

(a)

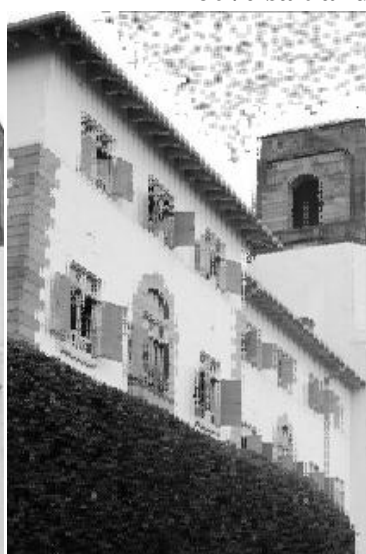

(b)

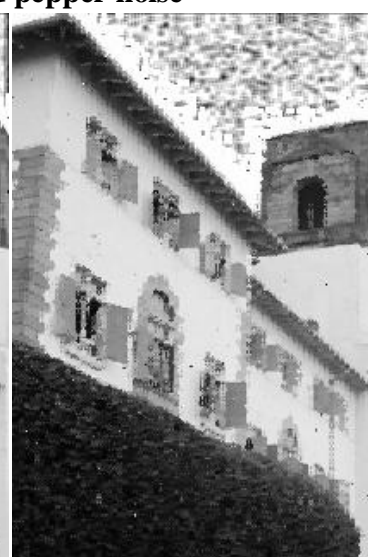

(c)

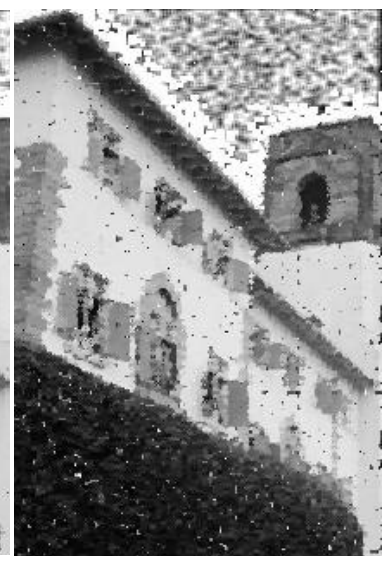

(d)

Figure 8: Application results of MDBUTMF on Makerere University Main Building (a) original image, (b) outcome of MDBUTMF for $20 \%$ salt and pepper noise, (c) outcome of MDBUTMF for $40 \%$ salt and pepper noise and (d) outcome of MDBUTMF for $60 \%$ salt and pepper noise 


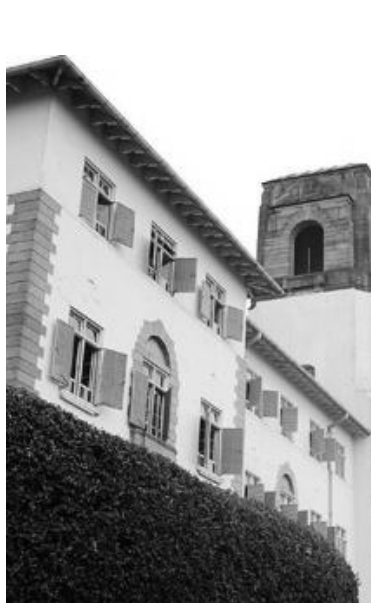

(a)

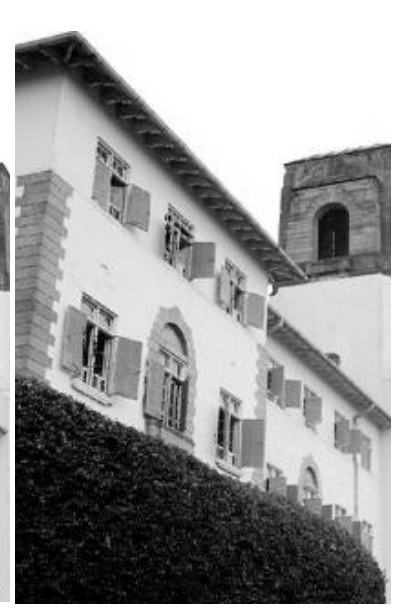

(b)

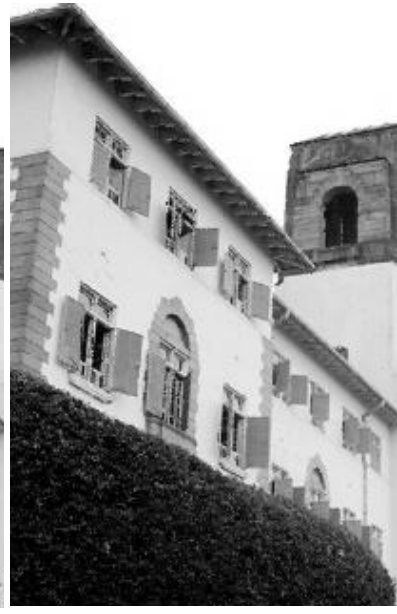

(c)

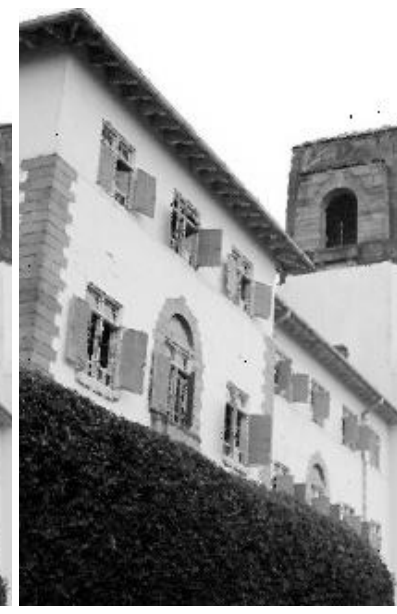

(d)

Figure 9: Application Result of WF-LMS-MDBUTMF on Noisy Makerere University Main Building Image: (a) original image, (b) outcome of WF-MDBUTMF for $20 \%$ salt \& pepper noise, (c) outcome of WF-MDBUTMF for $40 \%$ salt $\&$ pepper noise (d) outcome of WF-MDBUTMF for $60 \%$ salt \& pepper noise

The adaptive WF-LMS algorithm was implemented on the distorted image in figure 6 to de-noise the salt and pepper noise introduced to it. The outcome of this is shown in figure 7 which shows that the WF-LMS algorithm effectively removes white colour noise ( 250 valued) but fails to remove the black colour noise (zero valued). The undesired images in figures 7(b)-7(d) are a result of un-denoised salt noise (black dots) on the images. The density of the salt noise increases with increasing noise variances as shown in figures 7(b)-7(d). Therefore, the Weiner Filter is not good for salt and pepper noise at high noise variances.

The adaptive MDBUTMF algorithm of a $3 \mathrm{X} 3$ window size was implemented on the images in figure 6 . The outcome is shown in figure 8 . It is observed that the performance is better than the WF-LMS algorithm even at higher noise variances. However, the resolution of the image becomes very low as high noise variances are reached as shown in figure 8 (d). In addition, some artifacts have been introduced in the image. The algorithm effectiveness reduces as high noise variances are reached. In this study, the MDBUTMF begins to effectively fail to de-noise salt and pepper noise as the noise variance approaches $60 \%$.

In figure 9, the WF-LMS and MDBUTMF algorithms were combined. The combined algorithms were performed on the images in figure 6 in succession. The WF-LMS was first implemented followed by the MDBUTMF. The outcome turns out to be generally superior and it gives an image of relatively the same resolution as the original image irrespective of the noise level introduced in the signal. The advantage of this method is that, first the optimal filtering technique compares the corrupted pixels with the original image and adjusts the filter coefficients to minimize errors. Then, the pixels that get into MDBUTMF are approximated values to the original image. If this is not done before, the MDBUTMF would accept any value that had replaced the original pixel value due to decimation and translation. The remaining salt noises (zero valued) that were not de-noised by the WF-LMS are removed by conditions in the MDBUTMF algorithm. However, there are some few black dots in figure $9(\mathrm{c})$ caused by repeated substitution from some windows that contain only zeros. Increasing the window size of the MDBUTMF will help to reduce this problem.

The same procedure was repeated for the standard Lena picture as shown in figures 10 to 12 . The outcome was similar to the observations made for the image extracted from the Makerere University main building.

The process was also repeated on an image of the picture taken of the Department of Physics, Makerere University. These are shown in figures 13 to 15 . The observations made are found similar to the previous two images.

\subsection{Application to Lena Picture}

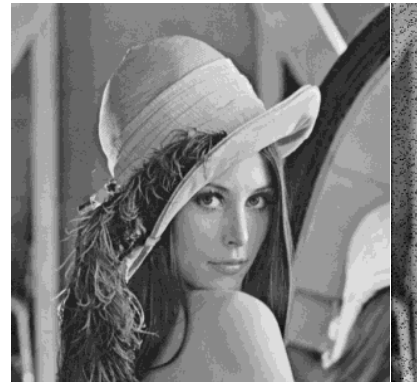

(a)

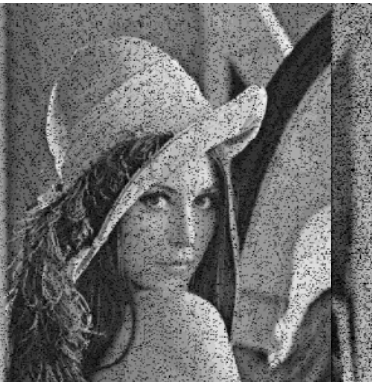

(b)

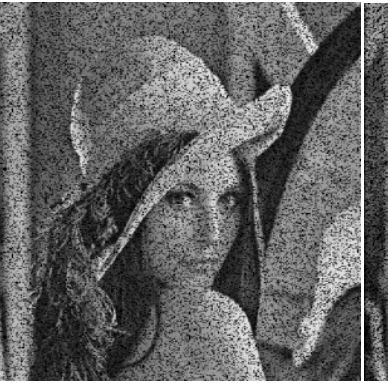

(c)

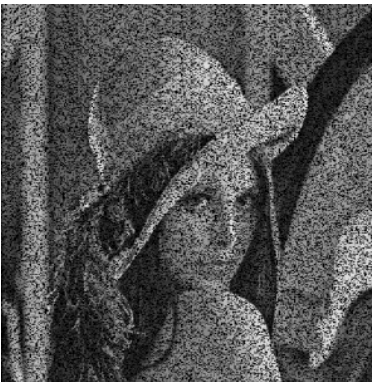

(d)

Figure 10: Application result of WF in de-noising salt and pepper noise from Lena: (a) original image, (b) outcome of WF for $20 \%$ salt and pepper noise, (c) outcome of WF for $\mathbf{4 0 \%}$ salt and pepper noise and (d) outcome of WF for $60 \%$ salt and pepper noise 


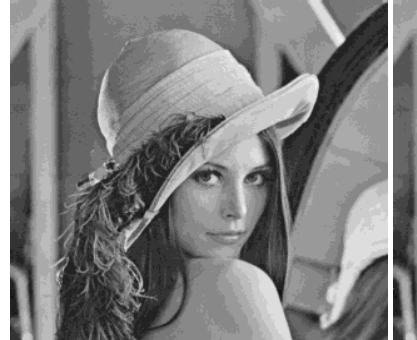

(a)

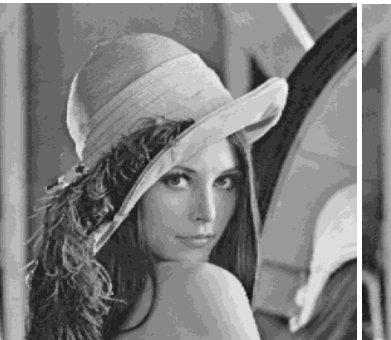

(b)

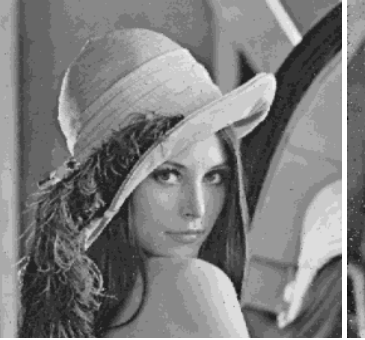

(c)

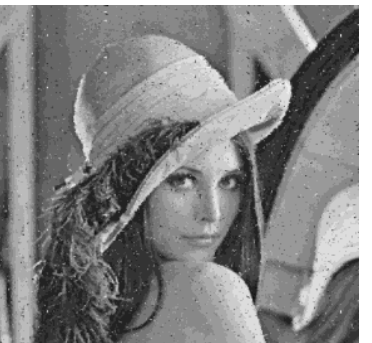

(d)

Figure 11: Application of MDBUTMF in de-noising Salt and pepper from Lena: (a) original image, (b) outcome of MDBUTMF for $20 \%$ salt and pepper noise, (c) outcome of MDBUTMF for $40 \%$ salt and pepper noise (d) outcome of MDBUTMF for $60 \%$ salt and pepper noise

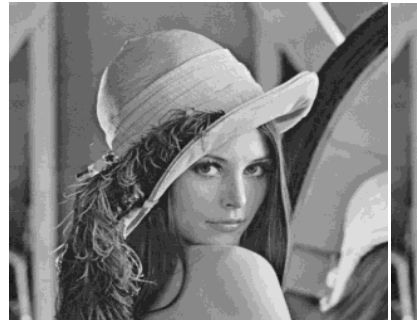

(a)

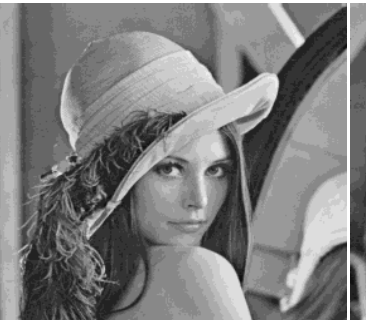

(b)

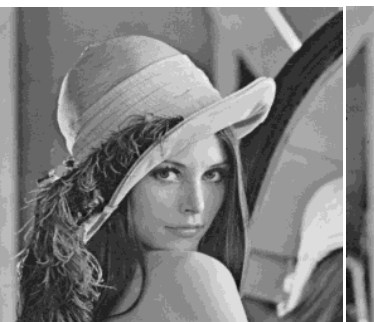

(c)

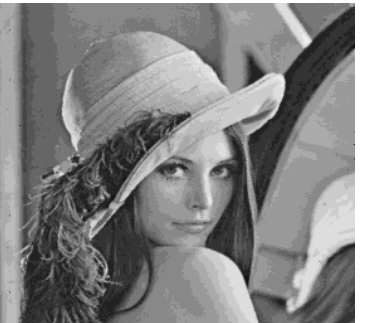

(d)

Figure 12: Application of WF-LMS-MDBUTMF in de-noising salt and pepper noise from Lena (a) original image, (b) outcome of WF-MDBUTMF for $20 \%$ salt \& pepper noise, (c) outcome of WF-MDBUTMF for $40 \%$ salt \& pepper noise (d) outcome of WF-MDBUTMF for $60 \%$ salt \& pepper noise

\subsection{Application to Makerere university-Department of physics Photo}

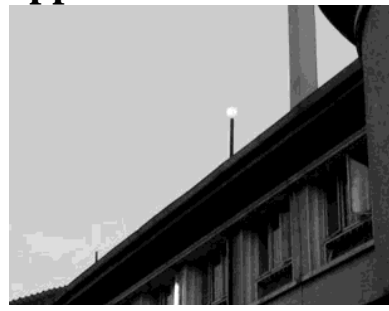

(a)

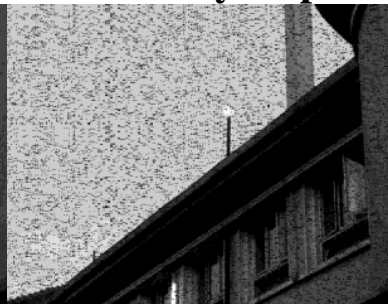

(b)

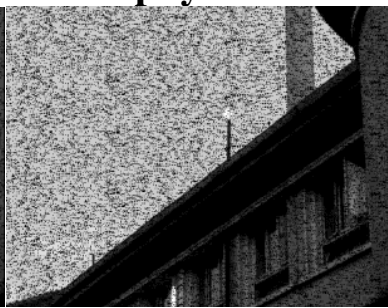

(c)

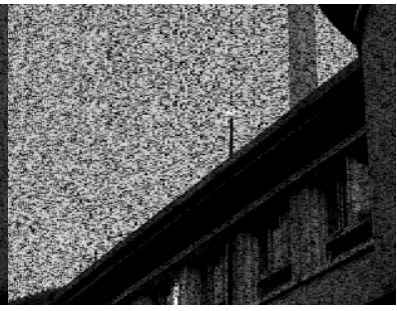

(d)

Figure 13: Application of WF in de-noising salt and pepper noise from physics department (a) original image, (b) outcome of WF for $20 \%$ salt and pepper noise, (c) outcome of WF for $40 \%$ salt and pepper noise and (d) outcome of WF for $60 \%$ salt and pepper noise

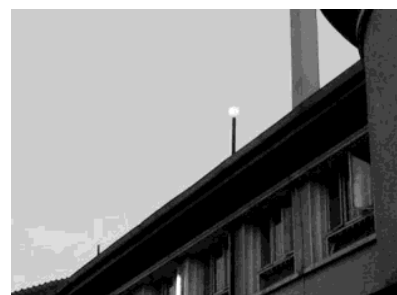

(a)

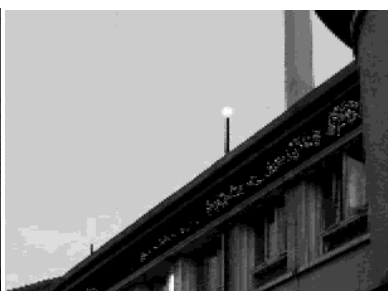

(b)

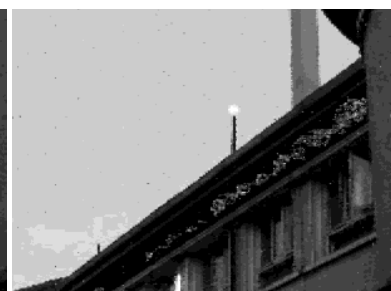

(c)

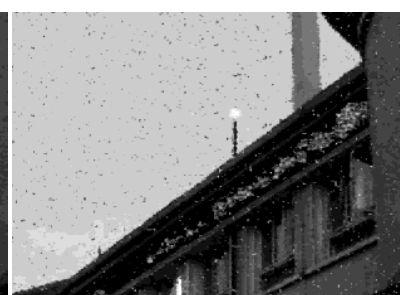

(d)

Figure 14: Application of MDBUTMF in de-noising salt and pepper noise from physics department: (a) original image, (b) outcome of MDBUTMF for $20 \%$ salt \& pepper noise, (c) outcome of MDBUTMF for $40 \%$ salt \& pepper noise and (d) outcome of MDBUTMF for $60 \%$ salt \& pepper noise.

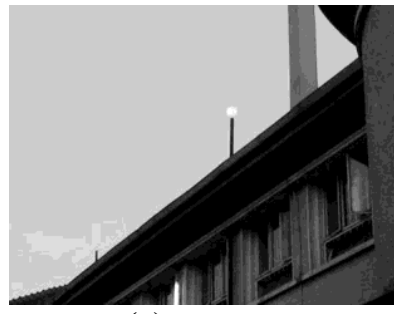

(a)

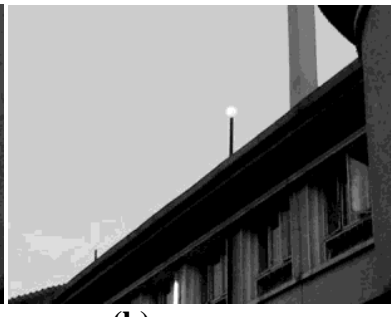

(b)

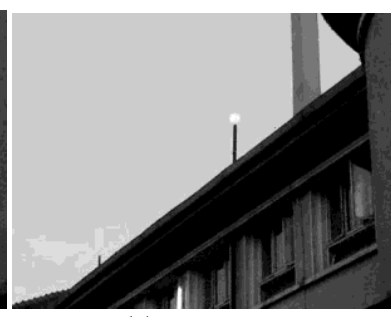

(c)

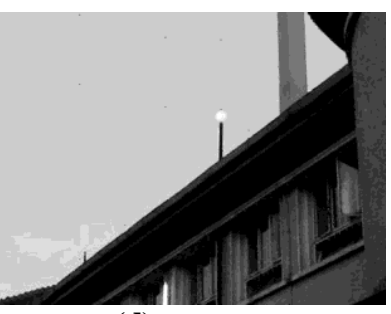

(d)

Figure 15: Application of the WF-LMS-MDBUTMF in de-noising salt and pepper noise from physics department (a) original image, (b) outcome of WF-MDBUTMF for $20 \%$ salt \& pepper noise, (c) outcome of WF-MDBUTMF for $40 \%$ salt \& pepper noise and (d) outcome of WF-MDBUTMF for $60 \%$ salt \& pepper noise 
Therefore, as can be seen from figures 9, 12 and 15, the proposed method performs better than the WF-LMS and the MDBUTMF in reconstructing the image blurred with salt and pepper noise. The method is able to reconstruct image from an image with salt and pepper noise variance as high as $90 \%$. The method has effective image restoration and visibility.

The MDBUTMF restores noisy image better than the WFLMS but it begins to smear the salt and pepper noise as the noise variances reach $60 \%$ as can be seen in figures $8(\mathrm{~d})$, 11(d) and 14(d). In addition, some artifacts get introduced into the image as in figures 8 and 14. These confirm that WF-LMS is ineffective at high salt and pepper noise variances ranging from $60 \%$ to $95 \%$ as shown in figures 8, 11 and 14 .
Our results confirmed that the WF-LMS would have been the best filter since it optimizes errors in the signal. Its failure is majorly attributed to its selective response to the specific salt and pepper noise. The selective response of WF-LMS to salt and pepper noise demands explanation which according to our understanding has not been revealed in the available literature.

\subsection{Quantitative Evaluation}

The performance evaluation of the filters was carried out using the MAE, MSE, PSNR and SNR. These parameters were evaluated for each of the techniques. The output of the proposed technique was compared with different standard techniques such as WF-LMS and MDBUTMF.

Table 2: Mean Absolute Error (MAE)

\begin{tabular}{|c|c|c|c|c|c|c|c|c|c|c|}
\hline \multirow[t]{3}{*}{$\overline{\mathrm{NV}}$} & \multirow{3}{*}{$\begin{array}{l}\text { Image } \\
\text { status }\end{array}$} & \multicolumn{9}{|c|}{ Mean Average Error (MAE) } \\
\hline & & \multicolumn{3}{|c|}{ Muk main building } & \multicolumn{3}{|l|}{ Lena } & \multicolumn{3}{|c|}{ Dep. Of Physics } \\
\hline & & WF & $\begin{array}{l}\text { MDBUT } \\
\text { MF }\end{array}$ & $\begin{array}{l}\text { WF- } \\
\text { MDBUT } \\
\text { MF }\end{array}$ & WF & $\begin{array}{l}\text { MDBUT } \\
\text { MF }\end{array}$ & $\begin{array}{l}\text { WF- } \\
\text { MDBUT } \\
\text { MF }\end{array}$ & WF & $\begin{array}{l}\text { MDBUT } \\
\text { MF }\end{array}$ & $\begin{array}{l}\text { WF- } \\
\text { MDBUT } \\
\text { MF }\end{array}$ \\
\hline \multirow[t]{2}{*}{0.2} & Noisy & \multicolumn{3}{|l|}{27.0537} & \multicolumn{3}{|l|}{27.0503} & \multicolumn{3}{|l|}{27.0537} \\
\hline & Denoised & 17.9616 & 9.3264 & 3.1787 & 12.9272 & 2.5268 & 0.7702 & 13.9324 & 2.9838 & 1.5178 \\
\hline \multirow[t]{2}{*}{0.4} & Noisy & \multicolumn{3}{|l|}{54.7266} & \multicolumn{3}{|l|}{52.3389} & \multicolumn{3}{|l|}{52.6865} \\
\hline & Denoised & 26.1669 & 13.4489 & 3.5493 & 25.0201 & 3.3740 & 1.1094 & 26.1649 & 3.9561 & 1.4891 \\
\hline \multirow[t]{2}{*}{0.6} & Noisy & \multicolumn{3}{|l|}{78.5960} & \multicolumn{3}{|l|}{77.2506} & \multicolumn{3}{|l|}{77.1056} \\
\hline & Denoised & 38.3135 & 19.9013 & 3.9348 & 37.4933 & 5.9865 & 1.5283 & 38.3135 & 6.5899 & 1.4926 \\
\hline
\end{tabular}

Table 3: Mean Square Error (MSE)

\begin{tabular}{|c|c|c|c|c|c|c|c|c|c|c|}
\hline \multirow[t]{3}{*}{ NV } & \multirow{3}{*}{$\begin{array}{l}\text { Image } \\
\text { status }\end{array}$} & \multicolumn{9}{|c|}{ Mean Squire Error (MSE) } \\
\hline & & \multicolumn{3}{|c|}{ Muk main building } & \multicolumn{3}{|l|}{ Lena } & \multicolumn{3}{|c|}{ Dep. Of Physics } \\
\hline & & WF & $\begin{array}{l}\text { MDBUT } \\
\text { MF }\end{array}$ & $\begin{array}{l}\text { WF- } \\
\text { MDBUT } \\
\text { MF }\end{array}$ & WF & $\begin{array}{l}\text { MDBUT } \\
\text { MF }\end{array}$ & $\begin{array}{l}\text { WF- } \\
\text { MDBUT } \\
\text { MF }\end{array}$ & WF & $\begin{array}{l}\text { MDBUTM } \\
\mathrm{F}\end{array}$ & $\begin{array}{l}\text { WF- } \\
\text { MDBUT } \\
\text { MF }\end{array}$ \\
\hline \multirow[t]{2}{*}{20} & Noisy & \multicolumn{3}{|l|}{5214.6} & \multicolumn{3}{|l|}{3725.2645} & \multicolumn{3}{|l|}{4735.6571} \\
\hline & Denoised & 3097.3 & 494.25 & 58.5533 & 1777.8706 & 47.4572 & 4.2685 & 2300.0119 & 192.7102 & 14.935 \\
\hline \multirow[t]{2}{*}{40} & Noisy & \multicolumn{3}{|l|}{9818.5} & \multicolumn{3}{|l|}{7458.5628} & \multicolumn{3}{|l|}{9496.5892} \\
\hline & Denoised & 5929.1 & 813.93 & 69.3635 & 3506.4011 & 76.1616 & 9.6328 & 4569.1684 & 253.7879 & 15.245 \\
\hline \multirow[t]{2}{*}{60} & Noisy & \multicolumn{3}{|c|}{14397.9} & \multicolumn{3}{|c|}{11131.5919} & \multicolumn{3}{|l|}{14008.0184} \\
\hline & Denoised & 8737.5 & 1585.05 & 103.138 & 5300.983 & 259.090 & 20.811 & 6835.0038 & 525.1168 & 19.188 \\
\hline
\end{tabular}

Table 4: Peak Signal to Noise Ratio (PSNR)

\begin{tabular}{|c|c|c|c|c|c|c|c|c|c|c|}
\hline \multirow[t]{3}{*}{$\mathrm{NV}$} & \multirow{3}{*}{$\begin{array}{l}\text { Image } \\
\text { status }\end{array}$} & \multicolumn{9}{|c|}{ Peak signal to noise ratio (PSNR $(\mathrm{dB}))$} \\
\hline & & \multicolumn{3}{|c|}{ Muk main building } & \multicolumn{3}{|l|}{ Lena } & \multicolumn{3}{|c|}{ Dep. Of Physics } \\
\hline & & WF & $\begin{array}{l}\text { MDBUT } \\
\text { MF }\end{array}$ & $\begin{array}{l}\text { WF- } \\
\text { MDBUT } \\
\text { MF }\end{array}$ & WF & $\begin{array}{l}\text { MDBUT } \\
\text { MF }\end{array}$ & $\begin{array}{l}\text { WF- } \\
\text { MDBUT } \\
\text { MF }\end{array}$ & WF & $\begin{array}{l}\text { MDBUT } \\
\text { MF }\end{array}$ & $\begin{array}{l}\text { WF- } \\
\text { MDBUT } \\
\text { MF }\end{array}$ \\
\hline \multirow[t]{2}{*}{20} & Noisy & \multicolumn{3}{|l|}{10.9586} & \multicolumn{3}{|l|}{12.4192} & \multicolumn{3}{|l|}{13.2982} \\
\hline & Denoised & 13.221 & 21.191 & 30.4553 & 15.6318 & 31.3678 & 41.8280 & 14.5135 & 25.2817 & 36.3885 \\
\hline \multirow[t]{2}{*}{40} & Noisy & \multicolumn{3}{|l|}{8.2104} & \multicolumn{3}{|l|}{9.4043} & \multicolumn{3}{|l|}{8.3551} \\
\hline & Denoised & 10.401 & 17.216 & 25.9034 & 12.6822 & 29.3134 & 38.2933 & 11.5324 & 24.0860 & 36.2994 \\
\hline \multirow[t]{2}{*}{60} & Noisy & \multicolumn{3}{|l|}{6.5478} & \multicolumn{3}{|l|}{7.6652} & \multicolumn{3}{|l|}{6.6670} \\
\hline & Denoised & 8.716 & 16.130 & 27.9966 & 10.8872 & 23.9962 & 34.9478 & 9.7834 & 20.9278 & 35.3005 \\
\hline
\end{tabular}


Table 5: Signal to Noise Ratio (SNR)

\begin{tabular}{|c|c|c|c|c|c|c|c|c|c|c|}
\hline NV & Image status & \multicolumn{9}{|c|}{ Signal to Noise Ratio $(\mathrm{SNR}(\mathrm{dB}))$} \\
\hline & & \multicolumn{3}{|c|}{ Muk main building } & \multicolumn{3}{|l|}{ Lena } & \multicolumn{3}{|c|}{ Dep. Of Physics } \\
\hline \multirow[t]{2}{*}{0.2} & Noisy & \multicolumn{3}{|l|}{17.0803} & \multicolumn{3}{|c|}{6.76230} & \multicolumn{3}{|l|}{6.8189} \\
\hline & Denoised & 22.2896 & 40.6420 & 61.9731 & 9.9749 & 25.7109 & 36.1711 & 9.9555 & 20.7237 & 31.8306 \\
\hline 0.4 & Noisy & \multicolumn{3}{|l|}{6.9241} & \multicolumn{3}{|c|}{3.74732} & \multicolumn{3}{|l|}{3.7971} \\
\hline 0.6 & Denoised & 11.9187 & 28.9887 & 56.3118 & 5.2303 & 18.3393 & 29.2909 & 5.2254 & 16.3698 & 30.7425 \\
\hline
\end{tabular}

From Tables 2 and 3, the results indicate that there is significant improvement in the quality of the de-noised signal in the order of WF-LMS, MDBUTMF and WF-MDBUTMF. WF-LMS algorithm produced the highest MAE and MSE meaning that noise is very dominant in the filter. The MDBUTMF algorithm produced lower MAE and MSE values compared to WF-LMS which gives the impression that the MDBUTMF significantly suppresses noise better than the WF-LMS even at high noise variance.

The proposed technique on the other hand has a strong capability to suppress noise compared to WF-LMS and MDBUTMF. From table 2, the MAE for Lena at $20 \%$ noise variance is $12.9272,2.5268$ and 0.7702 while the MSE for Lena at the same noise variance is $1777.8706,47.4572$ and 4.2685. Therefore, the performance of the filters increases in the order of WF-LMS, MDBUTMF and WF-MDBUTMF.

The PSNR and SNR were computed to quantify the amount of noise in the signal/image. The results are shown in tables 4 and 5. It is evident from Tables 4 and 5 that the proposed method performs best in terms of the PSNR and SNR, that is, it has higher PSNR than WF-LMS and MDBUTMF. Experimental results obtained show that at higher noise variance, the proposed method restores the original image much better than the WF-LMS and MDBUTMF when used separately. At noise variance of $40 \%$, the PSNR and the SNR of the restored Lena image improves by approximately $28 \mathrm{~dB}$ as compared to the noisy image as opposed to the case for the WF-LMS and MDBUTMF that give $3 \mathrm{~dB}$ and $20 \mathrm{~dB}$ respectively.

For quantitative and qualitative interpretation and graphical illustration, salt and pepper noise variances were expanded from $10 \%$ to $90 \%$ and Lena picture was used (tables 6 and 7). Primarily, the extension of the noise variances to the higher domain was to test the capability of the proposed method in de-noising signals at such domain.

From table 6, the noisy signal/image has high MAE and MSE values. When de-noised using the WF-LMS and the MDBUTMF, the noise is still dominant in the system as evidenced by the high MAE and MSE. However, the MDBUTMF proved to be better than then WF-LMS since it has lower MAE and MSE compared to the WF-LMS. The proposed method is however very effective in removing salt and pepper noise. This method has the lowest MAE and MSE. The plots of MAE and MSE against noise variances (\%) are graphically represented in figure 16 .

Table 6: MAE and MSE for WF, MDBUTMF and the WF-MDBUTMF for Lena for $10 \%-90 \%$ of salt and pepper noise variances

\begin{tabular}{|c|c|c|c|c|c|c|c|}
\hline \multicolumn{8}{|c|}{ Lena Picture } \\
\hline & & \multicolumn{3}{|l|}{ MAE } & \multicolumn{3}{|l|}{ MSE } \\
\hline & & WF & MDBUTMF & $\begin{array}{l}\text { WF- } \\
\text { MDBUTMF }\end{array}$ & WF & MDBUTMF & $\begin{array}{l}\text { WF- } \\
\text { MDBUTMF }\end{array}$ \\
\hline \multirow[t]{2}{*}{10} & Noisy & \multicolumn{3}{|l|}{14.6433} & 1891.3046 & & \\
\hline & De-noised & 6.6150 & 2.2648 & 0.6355 & 869.6618 & 43.0741 & 2.2978 \\
\hline \multirow[t]{2}{*}{20} & Noisy & \multicolumn{3}{|l|}{27.0503} & 3725.2645 & 3725.2645 & 3725.2645 \\
\hline & De-noised & 12.9272 & 2.5268 & 0.7702 & 1777.8706 & 47.4572 & 4.2685 \\
\hline \multirow[t]{2}{*}{30} & Noisy & \multicolumn{3}{|l|}{39.6230} & 5589.0534 & & \\
\hline & De-noised & 18.8571 & 2.8693 & 0.9205 & 2632.1154 & 56.2588 & 6.1451 \\
\hline \multirow[t]{2}{*}{40} & Noisy & \multicolumn{3}{|l|}{52.3389} & 7458.5628 & 7458.5628 & 7458.5628 \\
\hline & De-noised & 25.0201 & 3.3740 & 1.1094 & 3506.4011 & 76.1616 & 9.6328 \\
\hline \multirow[t]{2}{*}{50} & Noisy & 64.6079 & 64.6079 & 64.6079 & 9271.2559 & & \\
\hline & De-noised & 31.2975 & 4.1920 & 1.2995 & 4410.9503 & 125.2531 & 14.333 \\
\hline \multirow[t]{2}{*}{60} & Noisy & 77.2506 & 77.2506 & 77.2506 & 11131.5919 & 11131.592 & 11131.592 \\
\hline & De-noised & 37.4933 & 5.9865 & 1.5283 & 5300.9838 & 259.0900 & 20.8112 \\
\hline \multirow[t]{2}{*}{70} & Noisy & 89.8791 & & & 12979.9345 & & \\
\hline & De-noised & 43.7365 & 9.4617 & 1.7733 & 6184.1886 & 545.2034 & 28.6604 \\
\hline \multirow[t]{2}{*}{80} & Noisy & 102.4461 & & & 14867.1469 & & \\
\hline & De-noised & 50.0131 & 16.6491 & 2.1037 & 7098.6356 & 1197.4690 & 48.8479 \\
\hline \multirow[t]{2}{*}{90} & Noisy & 114.7627 & & & 16664.6946 & & \\
\hline & De-noised & 55.8937 & 30.4329 & 2.5342 & 7921.8781 & 2469.7822 & 79.9545 \\
\hline
\end{tabular}




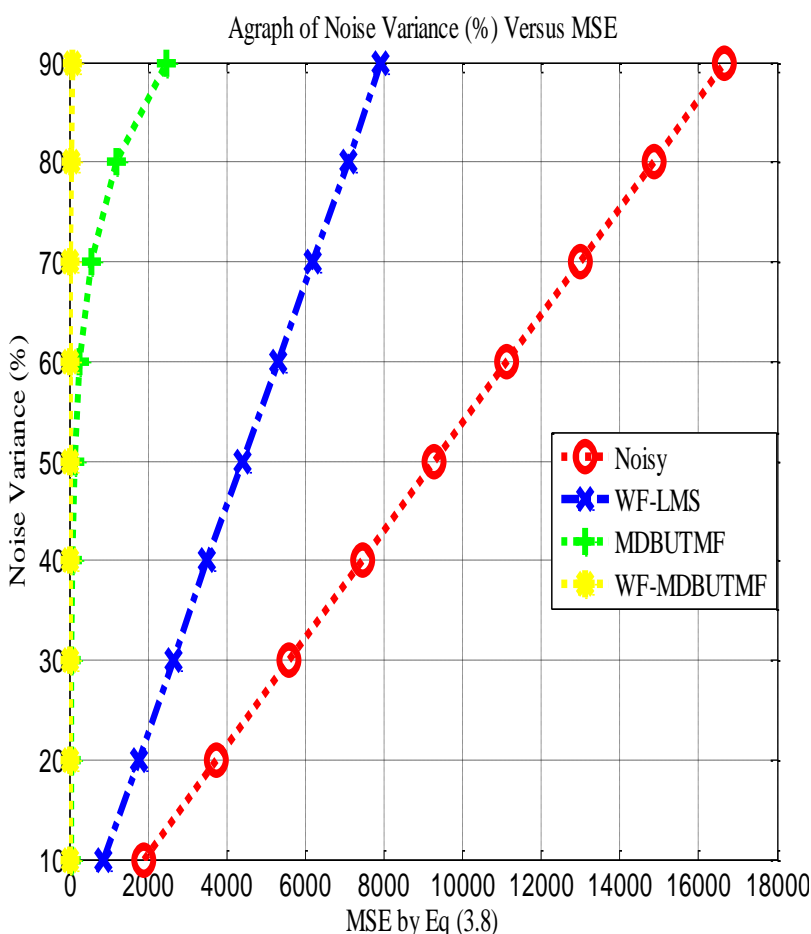

(a)

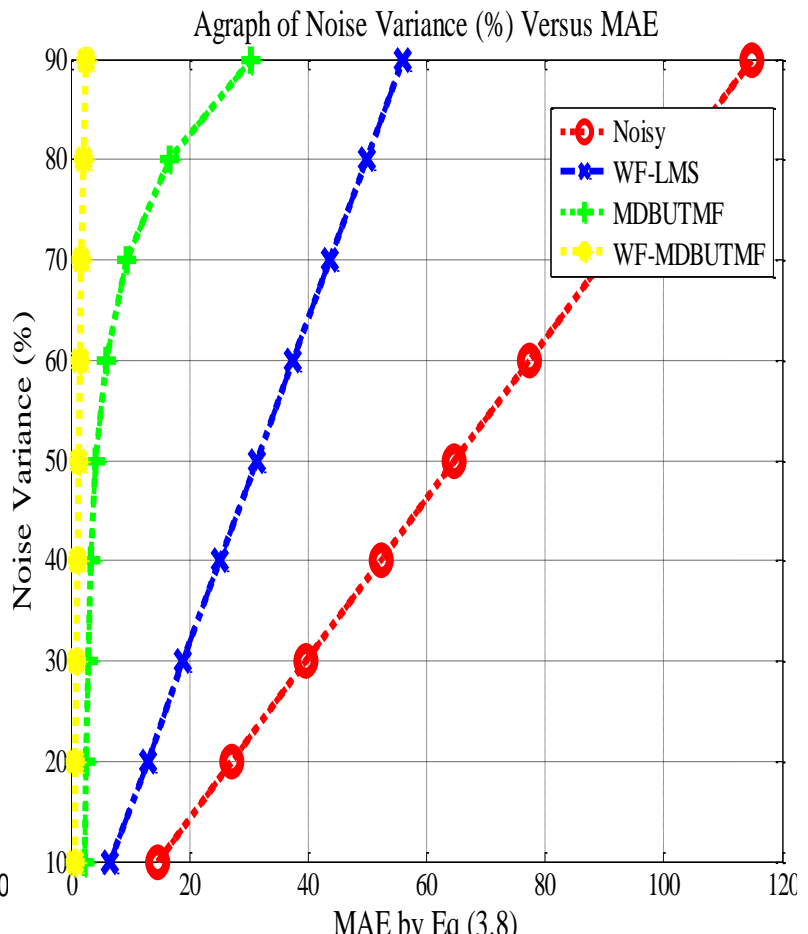

(b)

Figure 1: Graphical representation of MAE and MSE (a) Graph of MAE versus noise variances and (b) Graph of MSE versus noise variances

From figure 16, it shows that the proposed method has very low MAE and MSE compared to WF-LMS and MDBUTMF considered separately. Both MAE and MSE increase with increasing noise variances. The MAE and the MSE increase in the order of the WF-MDBUTMF, MDBUTMF, WF-LMS and the noisy signal/image as expected. This therefore shows that the WF-LMS and the MDBUTMF are vulnerable to noise reduction at high noise variances. MDBUTMF exhibits higher gradient for both the MAE and MSE. The MAE and the MSE for the MDBUTMF are almost constant at lower noise variances but it becomes non-linear as the noise variances reaches $60 \%$. It is an indication that the method becomes vulnerable when the noise reached 60 or $65 \%$.

Further quality analysis was considered using the PSNR and SNR. The computed PSNR and SNR were tabulated in table 7 and also graphically represented in figure 17 .

Table 7: PSNR, SNR for WF, MDBUTMF and the WF-MDBUTMF

\begin{tabular}{|c|c|c|c|c|c|c|c|}
\hline \multirow[b]{3}{*}{$\begin{array}{l}\mathrm{NV} \\
(\%)\end{array}$} & \multicolumn{7}{|l|}{ Lena Picture } \\
\hline & \multirow[b]{2}{*}{ Image status } & \multicolumn{3}{|c|}{ PSNR (dB) } & \multicolumn{3}{|c|}{ SNR (dB) } \\
\hline & & WF & MDBUTMF & $\begin{array}{l}\text { WF- } \\
\text { MDBUTMF }\end{array}$ & WF & MDBUTMF & $\begin{array}{l}\text { WF- } \\
\text { MDBUTMF }\end{array}$ \\
\hline \multirow[t]{2}{*}{10} & Noisy & 15.3631 & & & 9.7062 & & \\
\hline & De-noised & 18.7372 & 31.7886 & 44.5176 & 13.0804 & 26.1317 & 38.8607 \\
\hline \multirow[t]{2}{*}{20} & Noisy & 12.4192 & 12.4192 & 12.4192 & 6.7623 & & \\
\hline & De-noised & 15.6318 & 31.3678 & 41.8280 & 9.9749 & 25.7109 & 36.1711 \\
\hline \multirow[t]{2}{*}{30} & Noisy & 10.6574 & & & 5.0005 & & \\
\hline & De-noised & 13.9277 & 30.6289 & 40.2455 & 8.2708 & 24.9720 & 34.5886 \\
\hline \multirow[t]{2}{*}{40} & Noisy & 9.4043 & 9.4043 & 9.4043 & 3.7473 & & \\
\hline & De-noised & 12.6822 & 29.3134 & 38.2933 & 7.0253 & 23.6565 & 32.6364 \\
\hline \multirow[t]{2}{*}{50} & Noisy & 8.4594 & & & 2.8025 & & \\
\hline & De-noised & 11.6855 & 27.1529 & 36.5676 & 6.0286 & 21.4960 & 30.9107 \\
\hline \multirow[t]{2}{*}{60} & Noisy & 7.6652 & 7.6652 & 7.6652 & 2.0083 & & \\
\hline & De-noised & 10.8872 & 23.9962 & 34.9478 & 5.2303 & 18.3393 & 29.2909 \\
\hline \multirow[t]{2}{*}{70} & Noisy & 6.9981 & & & 1.3412 & & \\
\hline & De-noised & 10.2179 & 20.7652 & 33.5510 & 4.5610 & 15.1083 & 27.9010 \\
\hline \multirow[t]{2}{*}{80} & Noisy & 6.4085 & & & 0.7516 & & \\
\hline & De-noised & 9.6191 & 17.3481 & 31.2423 & 3.9621 & 11.6912 & 25.5854 \\
\hline \multirow[t]{2}{*}{90} & Noisy & 5.9128 & & & 0.2559 & & \\
\hline & De-noised & 9.1425 & 14.2042 & 29.1024 & 3.4856 & 8.5473 & 23.4454 \\
\hline
\end{tabular}


From table 7, high quality measure is obtained by the proposed method. The proposed method has higher PSNR and the SNR. The higher the PSNR or SNR, the better the image/signal quality. At $10 \%$ noise variance of salt and pepper, the PSNR are $18.7 \mathrm{~dB}, 31.7886 \mathrm{~dB}$ and $44.517 \mathrm{~dB}$ for the WF-LMS, MDBUTMF and the WF-MDBUTMF Agraph of Noise Variance (\%) Versus PSNR (dB)

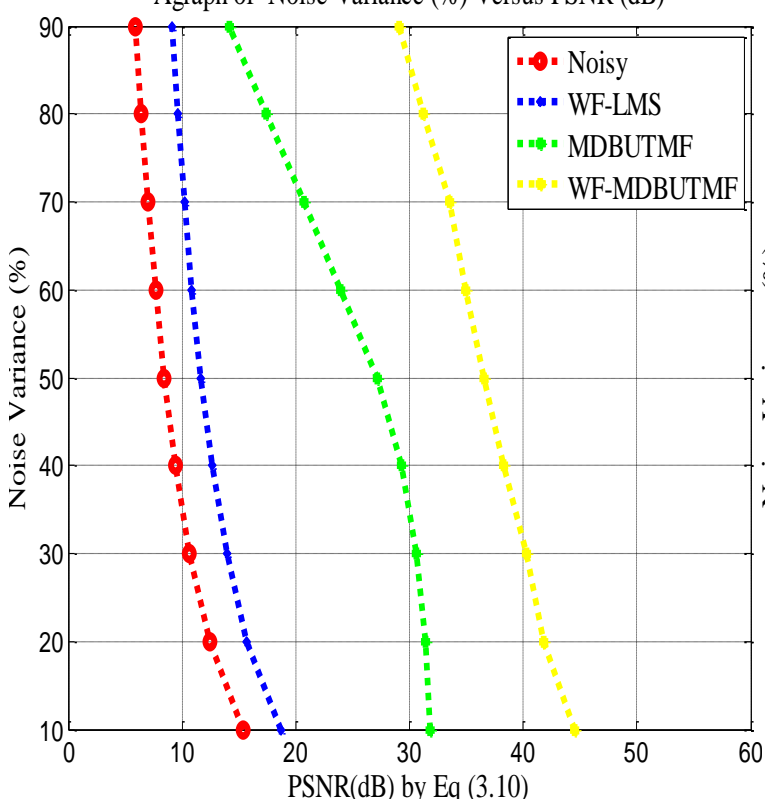

(a) respectively while that of SNR are $13.0804 \mathrm{~dB}, 26.1317 \mathrm{~dB}$ and $38.8607 \mathrm{~dB}$ respectively. Both the PSNR and the SNR increase in the order of the WF-LMS, the MDBUTMF and the proposed method (WF-MDBUTMF). The behavior of the PSNR and the SNR with change in noise variances is illustrated in figure 17.

Agraph of SNR (dB) Versus Noise Variance $(\%)$

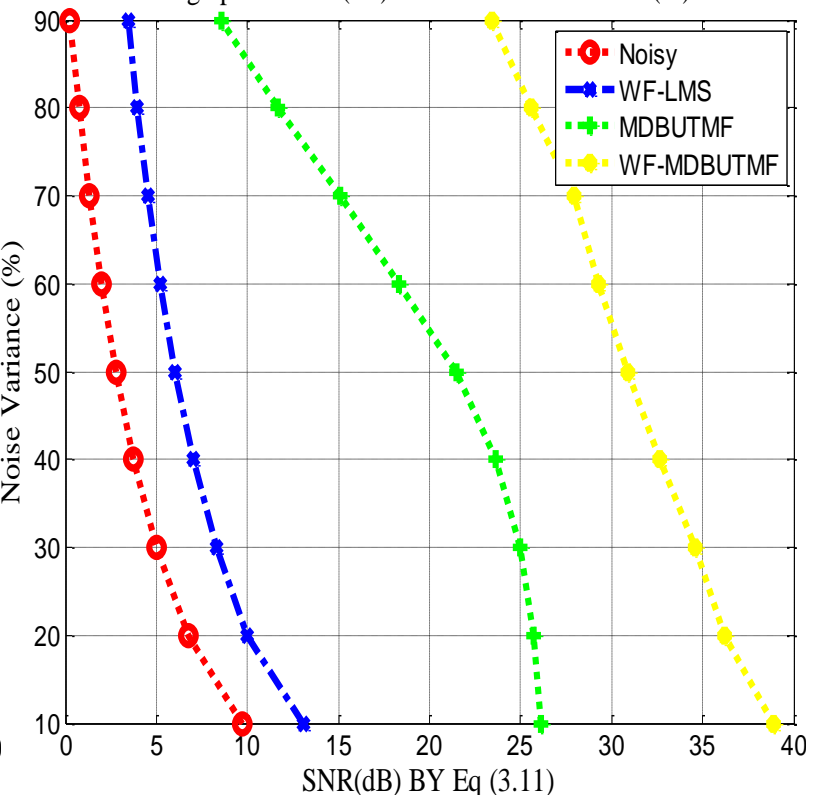

(b)

Figure 2: Graphical representation of PSNR and SNR (a) Graph of MAE versus noise variances and (b) Graph of MSE versus noise variances

From figures 17(a) and 17(b), the WF-LMS performs worst compared to MDBUTMF and the proposed method (WFMDBUTMF) in terms of PSNR and SNR. It exhibits a sharp decrease in the PSNR and the SNR and a non-linear characteristic as soon as the salt and pepper noise variance were increased from $10 \%$ to higher values. It also maintained lower PSNR and SNR which is a common characteristic of poor filters.

MDBUTMF exhibits a unique characteristic in terms of PSNR and SNR. The PSNR and the SNR remain high up to $40 \%$ of noise variance. From $40 \%$, there was a marked decrease in both the PSNR and the SNR. This therefore means that the MDBUTMF performs well at some level of low noise variance. However, the difference between the MDBUTMF and the WF-LMS in terms of the PSNR and the SNR at 90\% noise variance is only $5.0617 \mathrm{~dB}$. This means that even if the MDBUTMF is better than the WF-LMS, their performances are rather similar at high noise variances.

The proposed method (WF-MDBUTMF) maintained the highest PSNR and SNR well above the WF-LMS and the MDBUTMF. The difference between the PSNR and the SNR for the WF-MDBUTMF and those of the MDBUTMF and the WF-LMS at $10 \%$ noise variance is $25.7804 \mathrm{~dB}$ and $13.0514 \mathrm{~dB}$ respectively while that of SNR is $25.7803 \mathrm{~dB}$ and $13.0513 \mathrm{~dB}$ respectively.

\section{CONCLUSION}

Results of the proposed method (a combination of WF-LMS and MDBUTMF) show that its performance is better than the WF-LMS and the MDBUTMF in reconstructing the image blurred with salt and pepper noise. This proposed method has effective image restoration and visibility.
This new proposed method can further be improved by increasing the window size but care should be taken as increasing the window size could result into insertion of some artifacts in the image. This method could also be applied to a coloured image.

\section{REFERENCES}

[1] Huang, X. 1996. VLSI Implementation of Digital Adaptive Non-Linear Filter for Image Processing. A Thesis, Texas.

[2] Jyoti, G., Sandeep, M., Kavita, C., Deepika, \& Gard, A. 2011. De-speckling of Medical Ultrasound Images using Wiener Filter and Wavlet Transform. International Journal of Electronics \& Communication Technology, $2(3), 4$.

[3] Arastehfar, S., Pouyan, A. A., \& Jalalian, A. 2013. An enhanced median filter for removing noise from MR images. Journal of AI and Data Mining, 1(1), 5.

[4] Scrofani, J. W. 2005. Theory of Multirate Signal Pocessing with Application to Signal and Image Recnstruction. Monterey: Naval Posgraduate School.

[5] Abo-Zahhad, M. 2013. Current state and future directions of multirate filter banks and their applications. Digital Signal Processing , p. 24.

[6] Bojan, V. 2003. Multirate Signal Processing Concepts in Digital Communications. Pasadena: Bojan Vrcelj.

[7] Suresh, R. M., \& Kalavathy, S. 2011. A Switching Weighted Adaptive Median Filter for Impulse Noise Removal. International Journal of Computer Application (IJCA). 
[8] Kumar, S., Papendra, P., Gupta, M., \& Kumar, A. N. 2010. Performance Comparison of Median and Wiener Filter in Image De-noising. International Journal of Computer Applications , 5.

[9] Scott, C. D., \& Vijay, K. M. 1999. Introduction to Adaptive Filters. Boca Raton: CRC Press.

[10] Duadi, H., \& Zelevsky, Z. 2011. Wiener Filter in Gyrator Domain. Journal of Modern Optic, 58(18).

[11] Kiwata, H. 2012. Optimized Wiener Filter. Journal of Physics A: Mathematical and Theoretical(35), 118. Retrieved 2 11, 2014, from www.iopscience.iop.org/1751-8121/45/35/355101

[12] Kulkarni, P. R., Datar, A. V., \& Ajinkya, D. 2013. Performance Analysis of Different Inverse Filter Design Techniques. International Journal of Scientific Engineering and Technology, 2 (5), 7

[13] Gurmeet, K., \& Rupinder, K. 2012. Image De-noising Using Wavelet Transform and Various Filters. International Journal of Research in Computer Science, 2(2), 8. Retrieved 05 21, 2014, from www.ijorcs.org

[14] Gopi, K., Streeniv, A. R., \& Rajini, G. K. 2012. Removal of High Density Salt and Pepper Noise Through Modified Decision Based Unsymmetric Trimmed Median Filter. International Journal of Engineering Research and Applications (IJERA), 2(1), 5.
[15] Shyam, L., \& Mahesh, C. 2012. Efficient Algorithm for Enhancement of Images Corrupted by Salt \& and Pepper. WSEAS Transaction on Signal Processing, 8(3), 10.

[16] Harish, \& Gowtham, M. R. 2013. The Component Median Filter for Noise Removal in Digital Images. International Journal of Engineering Trends and Technology (IJETT) - Volume4,Issue5- May 2013, 4(5), 7. Retrieved $7 \quad 4, \quad 2014, \quad$ from http://www.ijettjournal.org/volume-4/issue-5/IJETTV4I5P102.pdf

[17] Chanchal, S., Saurabh, K. M., Pallavi, A., Mishra, G. R., \& Singh, O. P. 2013. Performance Comparison of Various Filters and Wavelet Transform for Image DeNoising. IOSR Journal of Computer Engineering (IOSRJCE), 10(1), 9.

[18] Gonzalez, C. R., Woods, E. R., \& Steven, L. E. 2009. Digital Image Processing Using MATLAB

[19] Semmlow, J. L. 2004. Biosignal and Biomedical signal Processing: Matlab Based Application. New York: Marcel Dekker, Inc.

[20] Sanker, R. V., Moorthy, K. V., Moonsoor, A. S., \& Begun, R. S. 2012. Split Weiner Filtering in Addaptive System. International Journal of Engineering Ressarch and Technology, 1(8). Retrieved 2 11, 2014, from www.ijert.org 